\title{
Evaluation of the Corneal Thickness and Endothelial Changes Following Uncomplicated Phacoemulsification in Diabetic and Non-Diabetic Patients by Pentacam and Specular Microscopy Motaz Bellah Abdel Hameed Erfan', Hossam El-Den Mohamed Khalii², Hazem Effat Haroun ${ }^{2}$, Ahmed Tharwat $\mathbf{A G}^{* 1}$ \\ ${ }^{1}$ Department of Ophthalmology, Faculty of Medicine, Misr University for Science and Technology \\ ${ }^{2}$ Department of Ophthalmology, Faculty of Medicine, Beni-Suef University \\ *Corresponding author: Ahmed Tharwat, Mobile: +201207782340, Email:a.teyeclinic @yahoo.com
}

\begin{abstract}
Background: Diabetes mellitus (DM) is an important health problem that induces unrestful complications. It causes significant morbidity owing to specific microvascular complications such as diabetic retinopathy, diabetic cataract, diabetic keratopathy, and diabetic optic nerve diseases.

Patients and Methods: This is a prospective, comparative case series study assessing the corneal endothelial status in 20 diabetics and 20 non diabetics before, after 1 day, 1 week and 1 month of uneventful phacoemulsification using phaco-chop technique. Central corneal thickness (CCT), as well as endothelial cell density (ECD), coefficient of variation $(\mathrm{CV})$ and percentage of polymegathism or hexagonality were measured by specular microscopy. The corneal thickness at 0,2 and $4 \mathrm{~mm}$ from center of the cornea and corneal volume at 3,5 and $7 \mathrm{~mm}$ from the center of the cornea. Changes were compared between both groups using pentacam in the same follow up period.

Results: Preoperative CCT was found to be significantly higher in diabetic than in non-diabetic group. ECD and CV were insignificantly higher in diabetics in contrast with the percentage of hexagonality, which was found to be insignificantly lower preoperatively. Diabetics showed insignificant higher loss in endothelial cell count as compared to non-diabetics. Furthermore, the diabetics showed a slower recovery trend in the endothelial healing response as evidenced by lower change in the CV\%. Postoperative CCT measurements demonstrated no significant variations between groups evidenced by specular microscopy as well as changes in corneal thickness and volume evidenced by pentacam.

Conclusion: The present study revealed no significant differences between preoperative and postoperative values neither in terms of mean ECD values, nor in corneal thickness and corneal volume between diabetic patients and nondiabetic patients undergoing phacoemulsification employing phaco-chop technique.

Keywords: Cataract Surgery, Corneal Thickness, Endothelial Changes, Specular Microscopy, Pentacam, Diabetes mellitus.
\end{abstract}

\section{INTRODUCTION}

The corneal endothelium has a vital role in the maintenance of corneal transparency. This is accomplished by its effectiveness in keeping the corneal stroma in a state of continuous dehydration through its two main actions; active fluid pump and barrier function. Any compromise in these activities has a direct effect on corneal transparency ${ }^{(\mathbf{1})}$.

The corneal endothelium is known to undergo damage during phacoemulsification ${ }^{(2)}$. The endothelial cell count is also known to decline with age ${ }^{(3)}$. With increase in life expectancy as most of the patients undergoing phacoemulsification belong to the elderly age group, endothelial damage during surgery becomes an important factor.

Diabetes mellitus (DM) is among the most prevalent and morbid chronic diseases affecting millions worldwide. According to the Global Burden of Disease report, the prevalence of diabetes rose from approximately 333 million in 2005 to approximately 435 million in $2015^{(4)}$. DM leads to various ocular complications such as diabetic retinopathy, diabetic cataract, diabetic keratopathy and diabetic optic nerve diseases. Diabetes mellitus also has been found to be detrimental to the corneal endothelium. Patients with diabetes have been found to have morphologically abnormal endothelium that is pleomorphism and polymegathism ${ }^{(5)}$. Thus, an elderly diabetic patient undergoing phacoemulsification is particularly vulnerable to greater endothelial damage during surgery. This hypothesis has been supported by few previous studies ${ }^{(6,7}$ and 8$)$ but still remains controversial. Inoue et al. ${ }^{(9)}$ in their study showed that presence of type 2 diabetes mellitus is irrelevant to any of the parameters of corneal endothelial cells in patients undergoing cataract surgery.

In a recent study, Storr- Paulsen et al. ${ }^{(10)}$ showed that type 2 diabetes has no impact on corneal cell density or morphology in participants with good glycemic status. In another study, it was reported that diabetes can affect both the corneal thickness and the corneal endothelium. Hyperglycemia alone can lead to endothelial alterations that can convert a routine surgery into a high-risk surgical case ${ }^{(7)}$.

\section{AIM OF THE WORK}

To evaluate the corneal thickness changes using pentacam and endothelial changes using specular microscopy in diabetic patients as compared to nondiabetics after uneventful phacoemulsification surgery. 


\section{PATIENTS AND METHODS}

This is a prospective, comparative, and interventional case series that included forty eyes of forty adult Egyptian patients aged between 45 and 65 years old, suffering from age-related nuclear cataract scheduled for phacoemulsification. It took place between November 2018 and July 2019. Patients were recruited from the Outpatient Clinic of the Ophthalmology Department of Beni-Suef University and Misr University for Science and Technology (M.U.S.T), Giza.

\section{Ethical approval:}

This study was approved by the Local Medical Ethics Committee with adherence to the guidelines of the declaration of Helsinki and a signed written consent was obtained from each patient.

All patients were subjected to complete ophthalmological examination including slit lamp examination, unaided and best corrected visual acuity using Snellen chart, fundus examination and Goldman applanation tonometry. Lens Opacities Classification System III (LOCS III) was used to grade the cataract. Specular microscopy (using Topcon SP-1P, Tokyo, Japan) and (Oculus HR Pentacam, Germany) were done to evaluate corneal status, preoperatively and at 1 day, 1 week and 1 month after surgery in all patients. Three readings were taken by specular microscopy and an average of three was finally taken as the result. Extreme variations in values were discarded. The measurements were done by a single operator to measure central corneal ECD (cells $/ \mathrm{mm}^{2}$ ), variation in size of corneal endothelial cells [percentage coefficient of variation $(\% \mathrm{CV})]$ and percentage of hexagonal cells and CCT (in micrometers). Pentacam was used before and 1 day, 1 week and 1 month after surgery to evaluate corneal thickness at 0,2 and $4 \mathrm{~mm}$ diameter and corneal volume at 3, 5 and 7-mm from center of the cornea.

Patients included in the study had grade 2-3 nuclear cataract with any additional cortical (C) and posterior sub capsular (P) according to lens opacity classification system III (LOCS III). Complicated cases, cases with local Eye disease other than cataract, cases with associated corneal pathology (i.e. cornea guttata) and cases with previous history of ocular trauma or surgery were excluded from the study. Also, patients who failed to complete follow up visits were excluded.
All patients were operated upon by a single well-experienced surgeon using phaco-chop technique with the same phacoemulsification equipment (Infiniti Vision System, Alcon Laboratories Inc., Fort Worth, TX, USA, 2014) at similar settings. Estimated fluid used in each case was between $125 \mathrm{cc}$ and $150 \mathrm{cc}$ at flow rate of $30-35 \mathrm{cc} / \mathrm{min}$ with infusion bottle height set at $110 \mathrm{~cm}$. A standard preoperative regimen included eye drops moxifloxacin hydrochloride $0.5 \%$ and nepafenac $0.1 \%$, one drop/ 8 h. 1 day before surgery. Besides, eye drops tropicamide $1 \%$ and phenylephrine hydrochloride $5.0 \%$, one drop every $15 \mathrm{~min} 1 \mathrm{~h}$ before surgery. Topical and peribulbar anesthesia with 5-6 ml lidocaine hydrochloride $2 \%$ and mepivacaine was given before surgery. In all patients, preoperative pupillary dilatation around 7-8 $\mathrm{mm}$ could be achieved. A $2.4 \mathrm{~mm}$ superotemporal self-sealing clear corneal incision was made just in front of the vascular arcades of the corneoscleral limbus using a calibrated $2.4 \mathrm{~mm}$ sharp keratome. Viscoelastic substance (hydroxypropyl methyl cellulose $2 \%$ ) was injected into the anterior chamber. After the capsulorhexis, hydro dissection followed by nucleus rotation, phacoemulsification was done by a single method, i.e. phaco-chop technique.

After emulsification of nuclear fragments, irrigation aspiration of residual cortical matter with proper removal of lens epithelial cells was done. A foldable single piece IOL was implanted inside the capsular bag. Removal of viscoelastic material was done and finally, the incision was hydrated using a 30-gauge cannula. No sutures were applied. Postoperative treatment included prednisolone acetate $1 \%$ in gradually reducing doses for 4 weeks, Ofloxacin $0.3 \% 5$ times for 2 weeks.

\section{Statistical analysis}

Statistical analysis was done using SPSS computer software package, version 20.0, 2006, Echo Soft Corporation, USA. Qualitative data was expressed as frequencies and percentages. Quantitative data was expressed as mean \pm SD for parametric data and median \pm interquartile range (IQR) for nonparametric data. Differences between groups were assessed through oneway analysis of variance (one-way ANOVA) for parametric data and Kruskal-Wallis test for nonparametric data. All tests were tailed and considered significant at $\mathrm{p}<0.05$ and highly significant at $\mathrm{p}<0.01$.

\section{RESULTS}

The demographic data of patients is mentioned in table (1). The mean age was 56.50 years in diabetics and 56.55 years in the non-diabetic group.

Table (1): Demographic data.

\begin{tabular}{|c|c|c|c|c|c|c|}
\hline & \multicolumn{4}{|c|}{ Groups } & \multirow{3}{*}{$P$ value } \\
\hline & & \multicolumn{2}{|c|}{ Diabetic } & \multicolumn{2}{|c|}{ Non diabetic } & \\
\hline & & Mean & SD & Mean & SD & \\
\hline \multicolumn{2}{|c|}{ Age } & 56.50 & 4.39 & 56.55 & 4.63 & 0.972 \\
\hline \multirow{2}{*}{ Gender } & $\mathbf{M}$ & 6 & $30.0 \%$ & 8 & $40.0 \%$ & \multirow{2}{*}{0.507} \\
\hline & $\mathbf{F}$ & 14 & $70.0 \%$ & 12 & $60.0 \%$ & \\
\hline
\end{tabular}


The preoperative specular microscopy evaluation showed that CCT was significantly higher in diabetic than nondiabetic patients with a $(\mathrm{P})$ value $\mathbf{0 . 0 1 5}$. The $\mathrm{ECD}, \mathrm{CV}$ were slightly higher in diabetic group than in non-diabetics and hexagonality percentage was slightly lower in diabetic than in non-diabetic patients but these values were statistically insignificant as shown in table (2).

The first postoperative day specular microscopy evaluation showed significant increase in CD in diabetic more than in non-diabetic and significant lower percentage of hexagonality $(\mathrm{P}=\mathbf{0 . 0 4 6} \& \mathbf{0 . 0 0 5}$ respectively) (Table 2).

The first postoperative week and first postoperative month specular microscopy evaluations showed no significant differences between both groups (table 2).

Table (2): Preoperative and postoperative specular microscopy findings.

\begin{tabular}{|c|c|c|c|c|c|}
\hline \multirow{2}{*}{$\begin{array}{c}\text { Specular microscopy } \\
\text { parameters }\end{array}$} & \multicolumn{2}{|c|}{ Diabetic group } & \multicolumn{2}{|c|}{ Non diabetic group } & \multirow{2}{*}{$\begin{array}{c}(\mathbf{P}) \\
\text { value }\end{array}$} \\
\hline & Mean & SD & Mean & SD & \\
\hline \multicolumn{6}{|c|}{ Central corneal thickness (CCT) } \\
\hline Preoperative & 515.20 & 33.55 & 492.85 & 19.95 & 0.015 \\
\hline Day one postoperative & 571.15 & 40.33 & 545.55 & 42.94 & 0.059 \\
\hline One week postoperative & 528.60 & 38.51 & 509.05 & 29.57 & 0.080 \\
\hline One month postoperative & 525.95 & 44.52 & 514.10 & 26.73 & 0.314 \\
\hline \multicolumn{6}{|c|}{ Endothelial cell density (ECD) } \\
\hline Preoperative & 2901.35 & 382.83 & 2845.90 & 310.17 & 0.618 \\
\hline Day one postoperative & 3223.55 & 395.91 & 2981.05 & 344.05 & 0.046 \\
\hline One week postoperative & 2822.55 & 459.60 & 2779.45 & 358.08 & 0.743 \\
\hline One month postoperative & 2661.20 & 399.95 & 2874.10 & 387.11 & 0.095 \\
\hline \multicolumn{6}{|c|}{ Coefficient of variation $(\mathrm{CV})$} \\
\hline Preoperative & 40.30 & 3.61 & 38.35 & 5.61 & 0.199 \\
\hline Day one postoperative & 42.90 & 3.13 & 41.35 & 6.86 & 0.364 \\
\hline One week postoperative & 39.95 & 5.18 & 40.70 & 6.04 & 0.676 \\
\hline One month postoperative & 41.60 & 4.33 & 40.90 & 4.47 & 0.618 \\
\hline \multicolumn{6}{|c|}{ Hexagonality (HEX) } \\
\hline Preoperative & 43.65 & 7.59 & 47.05 & 6.95 & 0.148 \\
\hline Day one postoperative & 37.60 & 4.36 & 43.20 & 7.16 & 0.005 \\
\hline One week postoperative & 45.10 & 6.65 & 44.75 & 8.25 & 0.883 \\
\hline One month postoperative & 42.80 & 6.77 & 44.70 & 5.71 & 0.344 \\
\hline
\end{tabular}

The preoperative pentacam evaluation (corneal thickness and volume) was slightly higher in the diabetic group than in non-diabetic group but the difference between them was statistically insignificant (Tables 3 and 4).

The first postoperative day: Pentacam evaluation (corneal thickness and volume) was higher in the diabetic group than in non-diabetic group but the difference between them was statistically insignificant except in corneal volume $5 \mathrm{~mm}$ and 7 $\mathrm{mm}(\mathrm{P}$ value $=0.010 \& 0.031$ respectively $)($ Tables 3 and 4$)$.

The first postoperative week and first postoperative month: Pentacam evaluations (corneal thickness and volume) were higher in the diabetic group than in the non-diabetic group but the difference between them was statistically insignificant (Tables 3 and 4).

Regarding intraoperative and post-operative complications: one eye had intra operative posterior capsular rent and vitreous loss (managed intraoperative with implantation of 3 piece foldable IOL with anterior vitrectomy), two eyes had moderate oedema that improved within 2 weeks (with medical treatment). Six patients were lost during the follow up visits. Patients with intra or postoperative complications as well as those who fail to complete the study during the follow up visits were excluded from the study and other patients were replaced. 
Table (3): Preoperative and postoperative corneal thickness evaluation by Pentacam

\begin{tabular}{|c|c|c|c|c|c|}
\hline \multirow{3}{*}{$\begin{array}{c}\text { Corneal } \\
\text { Thickness By } \\
\text { Pentacam }\end{array}$} & \multicolumn{4}{|c|}{ Group } & \multirow{3}{*}{$P$ value } \\
\hline & \multicolumn{2}{|c|}{ Diabetic Group } & \multicolumn{2}{|c|}{ Non diabetic Group } & \\
\hline & Mean & SD & Mean & SD & \\
\hline \multicolumn{6}{|c|}{ Preoperative } \\
\hline Thickness 0mm & 515.25 & 27.55 & 502.50 & 26.06 & 0.174 \\
\hline Thickness 2mm & 526.45 & 27.24 & 514.55 & 24.95 & 0.158 \\
\hline Thickness 4mm & 560.30 & 28.63 & 546.25 & 24.94 & 0.106 \\
\hline Thickness 6mm & 610.95 & 33.00 & 599.20 & 28.10 & 0.233 \\
\hline \multicolumn{6}{|c|}{ Day One Postoperative } \\
\hline Thickness 0mm & 558.10 & 37.97 & 543.00 & 37.55 & 0.214 \\
\hline Thickness $2 \mathrm{~mm}$ & 576.45 & 28.13 & 557.50 & 41.23 & 0.098 \\
\hline Thickness 4mm & 621.15 & 26.16 & 599.05 & 48.27 & 0.080 \\
\hline Thickness $6 \mathrm{~mm}$ & 683.65 & 24.83 & 670.75 & 53.30 & 0.333 \\
\hline \multicolumn{6}{|c|}{ One Week Postoperative } \\
\hline Thickness 0mm & 535.10 & 48.01 & 515.55 & 25.85 & 0.117 \\
\hline Thickness $2 \mathrm{~mm}$ & 547.20 & 48.04 & 528.55 & 26.13 & 0.135 \\
\hline Thickness 4mm & 583.50 & 48.98 & 564.50 & 29.01 & 0.144 \\
\hline Thickness 6mm & 642.85 & 50.85 & 626.30 & 31.17 & 0.222 \\
\hline \multicolumn{6}{|c|}{ One Month Postoperative } \\
\hline Thickness 0mm & 526.70 & 43.68 & 516.15 & 26.93 & 0.364 \\
\hline Thickness 2mm & 538.10 & 43.40 & 531.00 & 30.61 & 0.553 \\
\hline Thickness $4 \mathrm{~mm}$ & 572.75 & 44.65 & 561.00 & 31.01 & 0.340 \\
\hline Thickness 6mm & 626.25 & 48.13 & 617.55 & 31.30 & 0.502 \\
\hline
\end{tabular}

Table (4): Preoperative and postoperative corneal volume evaluation by Pentacam

\begin{tabular}{|c|c|c|c|c|c|}
\hline \multirow{3}{*}{$\begin{array}{c}\text { Corneal Volume By } \\
\text { Pentacam }\end{array}$} & \multicolumn{4}{|c|}{ Group } & \multirow{3}{*}{ P Valu } \\
\hline & \multicolumn{2}{|c|}{ Diabetic } & \multicolumn{2}{|c|}{ Non diabetic } & \\
\hline & Mean & SD & Mean & SD & \\
\hline \multicolumn{6}{|c|}{ Preoperative } \\
\hline Corneal vol. 3mm & 3.80 & 0.18 & 3.68 & 0.18 & 0.053 \\
\hline Corneal vol. 5mm & 11.19 & 0.58 & 10.85 & 0.49 & 0.057 \\
\hline Corneal vol. $7 \mathrm{~mm}$ & 24.13 & 1.32 & 23.47 & 1.05 & 0.090 \\
\hline \multicolumn{6}{|c|}{ Day One Postoperative } \\
\hline Corneal vol. 3mm & 4.23 & 0.19 & 4.08 & 0.36 & 0.085 \\
\hline Corneal vol. 5mm & 12.67 & 0.45 & 12.01 & 0.97 & 0.010 \\
\hline Corneal vol. $7 \mathrm{~mm}$ & 27.53 & 0.81 & 26.46 & 1.98 & $\mathbf{0 . 0 3 1}$ \\
\hline \multicolumn{6}{|c|}{ One Week Postoperative } \\
\hline Corneal vol. 3mm & 3.96 & 0.31 & 3.81 & 0.18 & 0.069 \\
\hline Corneal vol. 5mm & 11.76 & 0.89 & 11.30 & 0.56 & 0.054 \\
\hline Corneal vol. $7 \mathrm{~mm}$ & 25.58 & 1.90 & 24.73 & 1.24 & 0.102 \\
\hline \multicolumn{6}{|c|}{ One Month Postoperative } \\
\hline Corneal vol. 3mm & 3.92 & 0.30 & 3.80 & 0.23 & 0.181 \\
\hline Corneal vol. 5mm & 11.56 & 0.83 & 11.26 & 0.64 & 0.211 \\
\hline Corneal vol. $7 \mathrm{~mm}$ & 25.06 & 1.88 & 24.33 & 1.30 & 0.158 \\
\hline
\end{tabular}




\section{DISCUSSION}

In our study, we did not include patients with very hard nuclei (Grade IV and above) because of high cumulative dissipated energy (CDE) required with such hard cataracts. Both groups had no significant difference regarding the grading of nuclear density.

In our study, we observed a postoperative corneal endothelial cell loss at 1 week and 1 month in diabetic subjects with an average of $(2.7 \%$ and $8.27 \%$ respectively) compared to non-diabetic subjects with an average of (2.3\% and $1.02 \%$ respectively), with insignificant differences found when comparing ECD between groups, which coincides with Erika et al. ${ }^{(11)}$, Ganesan et al. ${ }^{(12)}$ and Gonzalez-Salinas et al. ${ }^{(13)}$. However, we noticed a significant increase in ECD in the first postoperative day in diabetic group more than in non-diabetic group, which was unique in our study, the only clue we had for this increment was that the increase in $\mathrm{CV}$ values cause a decrease in accuracy of specular microscopy, as Mc Carey et al. ${ }^{(14)}$ explained that an increase in polymegathism causes a decrease in the accuracy of the average cell area.

Coefficient of variation is an indicator of the uniformity of the size of endothelial cells. High values indicate high levels of pleomorphism. It indicates the activity of the repair and healing mechanism of the endothelium after an insult (1). Preoperative values were comparable in the diabetics and non-diabetic patients in our study (slightly lower in the latter group). A higher CV during follow up (as compared to preoperative values) was seen in both groups, the diabetic group showed a statistically insignificant lesser change as compared to the non-diabetic group. This demonstrates the slower and weaker recovery of endothelial cells in diabetics. The trend analysis, which revealed a slower rate of change in diabetics, confirms this finding. Similar observation was made by Sahu $\boldsymbol{e t}$ al. ${ }^{(1)}$. A decrease in the CV was found by Hugod et al. ${ }^{(15)}$ (33.2 at 3 months as compared to 33.7 preoperatively) and this change was not found to be statistically significant. Studies by other authors also demonstrated that the coefficient of variation slightly increased 1 day after operation, but did not significantly differ between the 2 groups ${ }^{(16,17)}$.

These results support the theory that the endothelium in diabetic patients has slower and poor healing recovery response as evidenced by lower change in the coefficient of variation so a slower recovery in corneal edema after surgery. The corneal endothelium in diabetic patients is under metabolic stress, and weaker against mechanical loads, such as cataract surgery, than that in non-diabetic subjects who at 1 month was able to lower the percentage of the endothelial loss compared to diabetics, and this is supported by Morikubo et al. ${ }^{(16)}$.

Normal corneal endothelial cells have a hexagonal shape forming a regular mosaic. After insult, this morphology is disturbed (pleomorphism) and the percent of hexagonal cells is reduced ${ }^{(18)}$. In our study, similar to Sahu et al. ${ }^{(1)}$ and Lee et al. ${ }^{\left({ }^{(1)}\right)}$ there was a decrease in percentage of hexagonal cells was seen in the diabetic as well as in the non-diabetic patients, but this change was not statistically different in comparison with each other in all postoperative measurements except one day post-operative with $\mathrm{p}=$ 0.005 , which is similar to Morikubo et al. ${ }^{(16)}$ study. However regarding diabetic group only we found a significant decline in the percentage of hexagonal cells, which is similar to Hugod et al. ${ }^{(15)}$ indicating a slower endothelial cell repair in diabetics, which coincides with Ganesan et al. ${ }^{(12)}$ as well.

When the endothelium is stabilized after a period of rearrangement, the $\mathrm{CV}$ and the hexagonality shift towards the preoperative status ${ }^{(15)}$. This totally matches with our results in the first day postoperative where highest increment happened and shifted near baseline after one month in diabetic and non-diabetic corneas.

CCT was used in our study as a surrogate marker for endothelial function status. This is because a wellfunctioning endothelium keeps the corneal stroma in a dynamic state of continuous deturgescence ${ }^{(\mathbf{1 5})}$.

Concerning postoperative changes, the increase in central corneal thickness was highest one day after operation in diabetic and non-diabetic groups, but recovered thereafter. This goes along with what Sahu et al. ${ }^{(1)}$ and Ali et al. ${ }^{\left({ }^{(9)}\right)}$ had found in their work with CCT measurements in diabetic patients in comparison with healthy participants. Another study done by Morikubo et al. ${ }^{(16)}$ reported increment in central corneal thickness 1 month after operation that was $0.04 \%$ in the non-diabetic group and $1.6 \%$ in the diabetic group, showing significantly poor recovery in the latter. Therefore, recovery of corneal edema after cataract surgery may be delayed in the cornea of diabetic patients, as the corneal thickness will not have returned to the preoperative level 1 month after surgery.

However in our study, Serial changes in the increase in the central corneal thickness after operation were observed compared to the preoperative thickness. Increases observed 1 day, 1 week, and 1 month after operation were $10 \%, 3.28 \%$, and $4.31 \%$, respectively in the non-diabetic group and $10.85 \%, 2.6 \%$, and $2.08 \%$ respectively, in the diabetic group, but statistically insignificant. The increase after 1 month was higher in the non-diabetic group than in the diabetic group, indicating poor recovery regarding thickness in the latter, which is in contrast to Morikubo et al. ${ }^{(16)}$. Therefore, recovery of corneal edema after cataract surgery may be delayed in the cornea of non-diabetic patients, as the corneal thickness will not have returned to the preoperative level 1 month after surgery. This may be attributed to the age factor as some corneas that showed delayed recovery of the edema were of higher age group. 
In Chen et al. ${ }^{(20)}$ study, they found that diabetic patients had poor corneal endothelial function as evidenced by the higher CCT during the 1 week and 1 month postoperatively compared with the non-diabetic group. The increase in CCT in the 1st postoperative day and a gradual decline in the values by the first week and first 1 month postoperatively could be attributed to the inflammatory score as Ganesan $\boldsymbol{e t} \boldsymbol{a l}$. (12) explained in their results that as the inflammation decreases the corneal edema also decreases, but the CCT did not reach the baseline values even by the end of 3 months. Furthermore, the corneal endothelium photographed for analysis belonged to the central cornea. Thus, the changes in morphology may be more toward the periphery which will go undetected with the technique used in the previous studies. Several studies have used specular microscopy to quantitatively and qualitatively assess the corneal damage associated with phacoemulsification by measuring the degree of decrease in the central corneal cell density ${ }^{(21,22,23}$ and 24).

This assessment includes measuring cell density and morphology in a limited corneal area (area of $<1$ $\mathrm{mm}^{2}$ ). Thus it may not be an appropriate method for evaluating damage to the entire cornea. Therefore, it is difficult to statistically evaluate the entire cornea. Unlike specular microscopy, pentacam allows for quick, easy and reliable demonstration of anterior segment changes before and after phacoemulsification surgery and IOL implantation and offers corneal volume measurement in a central $3,5,7 \mathrm{~mm}$ area of the cornea with numerical values obtained can be used for statistical assessment with high reliability and repeatability (25). Moreover Suzuki et al. ${ }^{(25)}$ and Melissa et al. ${ }^{(26)}$ have used Scheimpflug imaging to analyze corneal edema based on the corneal volume at different areas as damage to endothelial cells also leads to an increase in corneal volume.

Some authors preferred pentacam over the specular microscopy as CCT and corneal volume could be considered as indirect indicators of corneal endothelial function. However, it's a marker for endothelial pump and barrier function which can't be assessed by the specular microscopy ${ }^{(19 \& 27)}$.

In our study, we found that pentacam gave slightly higher values of CCT in both groups pre operatively than specular microscopy. Also, it was found that it $\mathrm{t}$ underestimated the corneal thickness slightly post operatively compared to specular microscopy. Our impression is that the pentacam gives more relevant information than specular microscopy after phacoemulsification surgery because it correlates better with the endothelial cell loss. Possibly, this may be due to better reproducibility of its measurements. This is supported in Lundberg et al. ${ }^{(28)}$ where they assessed it with Orbscan II but is not supported by Melissa et al. ${ }^{(26)}$ who reported a thicker pentacam corneal measurements postoperatively.
To our knowledge this is the first report to compare corneal thickness and volume changes after phacoemulsification between diabetic and nondiabetic by pentacam. We used the pentacam to focus on the short-term changes in CCT and $\mathrm{CV}$ after uneventful phacoemulsification (central corneal thickness at $0 \mathrm{~mm}, 2 \mathrm{~mm}, 4 \mathrm{~mm}, 6 \mathrm{~mm} \& \mathrm{CV}$ at 3, 5, $7 \mathrm{~mm}$ ) for each group.

In our study, we found statistically insignificant higher corneal thickness in diabetics preoperatively. Similar results were obtained by Altay et al. ${ }^{(29)}$ who used pentacam to evaluate corneal thickness measurements between 127 patients with DM, and 137 age and sex-matched healthy controls.

In our study the mean preoperative and postoperative day 1 central corneal thickness measurements were higher $(515.25 \mu \mathrm{m}$ and 558.10 $\mu \mathrm{m})$ in the diabetic group than in the non-diabetic group $(502.50 \mu \mathrm{m}$ and $543.00 \mu \mathrm{m})$ respectively. Statistical analysis showed that the differences between the preoperative and postoperative follow ups mean values did not differ significantly for either the diabetic or non-diabetic groups. In addition, the preoperative and postoperative 1 day, 1 week and 1 month values did not differ between the two groups, but corneal volume changes were lesser in healthy controls.

Comparing with the preoperative data, the changes in central corneal thickness were $2.42 \%$ in the diabetic group and $2.716 \%$ in the non-diabetic group at 1 month postoperative, which goes along with specular microscopy findings that non-diabetic corneas tend to get more thickness increment of insignificant value.

Corneal volume assessed for the $3 \mathrm{~mm}$ central corneal area (CV3) increased on postoperative day 1 in the diabetic and non-diabetic groups $(\mathrm{P}=0.085)$, but decreased in postoperative week one and month to near baseline. The CV5 and CV7 showed significant difference between the two groups in preoperative and postoperative day 1 in both with $\mathrm{P}$ value $=0.010,0.031$ respectively. But, one week and one month postoperative measurements decreased with CV5 reaching near preoperative value, but CV7 didn't. This indicates that although phacoemulsification affects both groups and lead to increment in the corneal volume of both groups, our study revealed no significant difference found in diabetic or healthy controls.

The CV increment that occurred following phacoemulsification originates from damage to the corneal endothelial cells, which play a key role in maintaining a non-hydrous state of the cornea. In addition, because of various factors, such as the swelling of the corneal matrix layer due to intraoperative heating, corneal edema might also occur. However, as edema of the entire cornea could not be confirmed, a quantitative assessment of the 
overall corneal edema by phacoemulsification was performed based on the corneal volume in different points.

To summarize, our study has found that preoperative CCT was found to be significantly higher in diabetic than in non-diabetic group. ECD and CV were insignificantly higher in diabetics in contrast to percentage of hexagonality, which was found to be insignificantly lower preoperatively. Diabetic patients showed a significantly higher loss of corneal endothelial density after phacoemulsification as compared to non-diabetic patients. Furthermore, a slower and poor healing response was seen in diabetics as evidenced by lesser changes in the \% CV. However, these morphometric parameters did not translate in functional deficits as noted by non-significant changes in CCT and equivalent visual gain as compared to nondiabetic patients.

\section{CONCLUSION}

The present study reveals no significant differences between preoperative and postoperative values neither in terms of mean ECD values, nor in corneal thickness and corneal volume between diabetic patients and nondiabetic patients undergoing phacoemulsification employing phaco-chop technique.

After uneventful phacoemulsification, both groups had initial rise in CCT due to the breakdown of corneal endothelial function caused by the surgical procedure, however diabetic subjects were more vulnerable to surgical trauma, but the difference between the two groups are too small to be distinguished. However, despite significant slower process of cell repair in diabetic subjects, it was evidenced that there was change of hexagonality percentage, higher loss of endothelial cells and increment of coefficient of variation observed in follow up visits, functional ocular status seemed unchanged as judged by CCT measured by specular and pentacam. Considering that the postop CCT is not different between groups, it may suggest that despite losing more cells and having higher $\mathrm{CV}$, the endothelium of diabetic patients is still able to function normally. Moreover, pentacamdetermined corneal thickness and volumes may be useful in assessing phacoemulsification causing corneal damage, as seen in peripheral cornea in diabetic patient, the corneal volume increases after surgery may be indicative of the degree of the endothelial damage.

Financial support: none.

Conflicts of interest: none.

\section{REFERENCES}

1. Sahu P, Das G, Agrawal S et al. (2017): Comparative evaluation of corneal endothelium in diabetic patients undergoing phacoemulsification. Middle East Afr J Ophthalmol., 24: 195-201.
2. Gonen T, Sever O, Horozoglu F et al. (2012): Endothelial cell loss: Biaxial small-incision torsional phacoemulsification versus biaxial small-incision longitudinal phacoemulsification. J Cataract Refract Surg., 38: 1918-24.

3. Wang Y, Xia Y, Liu X et al. (2012): Comparison of bimanual and micro-coaxial phacoemulsification with torsional ultrasound. Acta Ophthalmol., 90: 184-187.

4. Ali BA, Salman AG, Mohamad AA et al. (2018): Evaluation of central corneal thickness as measured by pentacam and retinal and choroidal thickness as measured by optical coherence tomography before and after uneventful cataract phacoemulsification in normal and diabetic patients without retinopathy. J Egypt Ophthalmol Soc., 111: 37-42.

5. Choo M, Prakash K, Samsudin A et al. (2010): Corneal changes in type II diabetes mellitus in Malaysia. Int J Ophthalmol., 3: 234-6.

6. Su DH, Wong TY, Wong WL et al. (2008): Diabetes, hyperglycemia, and central corneal thickness: The Singapore Malay eye study. Ophthalmology, 115: 964-80.

7. Mathew PT, David S, Thomas N (2011): Endothelial cell loss and central corneal thickness in patients with and without diabetes after manual small incision cataract surgery. Cornea, 30: 424-8.

8. Sudhir RR, Raman R, Sharma T (2012): Changes in the corneal endothelial cell density and morphology in patients with type 2 diabetes mellitus: A population-based study, Sankara Nethralaya diabetic retinopathy and molecular genetics study (SN-DREAMS, report 23). Cornea, 31:1119-22.

9. Inoue K, Tokuda Y, Inoue Y et al. (2002): Corneal endothelial cell morphology in patients undergoing cataract surgery. Cornea, 21:360-3.

10. Storr-Paulsen A, Singh A, Jeppesen $H$ et al. (2014): Corneal endothelial morphology and central thickness in patients with type II diabetes mellitus. Acta Ophthalmol., 92: 158-60.

11. Erika F, Zamora-Ortiz $\mathbf{R}$, Gonzalez-Salinas $\mathbf{R}$ (2019): Endothelial cell density changes in diabetic and nondiabetic eyes undergoing phacoemulsification employing phaco-chop technique. International Ophthalmology, 39 (8): 1735-1741.

12. Ganesan N, Srinivasan R, Babu K et al. (2019): Risk factors for endothelial cell damage in diabetics after phacoemulsification. Oman J Ophthalmol., 12: 94-98.

13. Gonzalez-Salinas $R$, Garza-Leon $M$, Saenz-deViteri M et al. (2018): Comparison of cumulative dissipated energy delivered by active-fluidic pressure control phacoemulsification system versus gravityfluidics. International Ophthalmology, 38 (5): 19071913.

14. Mc Carey B, Edelhauser H, Lynn M (2008): Review of corneal endothelial specular microscopy for FDA clinical trials of refractive procedures, surgical devices, and new intraocular drugs and solutions. Cornea, 27 (1): 1-16.

15. Hugod M, Storr-Paulsen A, Norregaard JC et al. (2011): Corneal endothelial cell changes associated with cataract surgery in patients with type 2 diabetes mellitus. Cornea, 30: 749-53. 
16. Morikubo S, Takamura Y, Kubo E et al. (2004): Corneal changes after small-incision cataract surgery in patients with diabetes mellitus. Arch Ophthalmol., 122: 966-9.

17. Lee JS, Lee JE, Choi HY et al. (2005): Corneal endothelial cell change after phacoemulsification relative to the severity of diabetic retinopathy. J Cataract Refract Surg., 31: 742-9.

18. Yee RW, Matsuda M, Schultz RO et al. (1985): Changes in the normal corneal endothelial cellular pattern as a function of age. Curr Eye Res., 4: 671-8.

19. Ali S, Burak B, Musa C et al. (2016): Evaluation of anterior segment parameter changes using the Sirius after uneventful phacoemulsification. Korean Journal of Ophthalmology, 30 (4): 251-257.

20. Chen D, Tang Q, Yu F et al. (2019): Consecutive drilling combined with phaco chop for full thickness segmentation of very hard nucleus in coaxial micro incisional cataract surgery. BMC Ophthalmol., 19 (1): 20-24.

21. Hayashi K, Hayashi H, Nakao F et al. (1996): Corneal endothelial cell loss in phacoemulsification surgery with silicone intraocular lens implantation. J Cataract Refract Surg., 22 (6): 743-747.

22. Kim E (1997): Corneal endothelial damage by air bubbles during phacoemulsification. Arch Ophthalmol., 115: 81-88.

23. Kim E, Cristol S, Kang S et al. (2002): Viscoelastic protection from endothelial damage by air bubbles. J Cataract Refract Surg., 28: 1047-1053.
24. Milla' E, Verge's C, Cipre's M (2005): Corneal endothelium evaluation after phacoemulsification with continuous anterior chamber infusion. Cornea, 24: 278 282.

25. Suzuki H, Oki K, Takahashi K et al. (2007): Functional evaluation of corneal endothelium by combined measurement of corneal volume alteration and cell density after phacoemulsification. J Cataract Refract Surg., 33: 2077-2082.

26. Melissa W, Shukla AN, Munir W (2014): Correlation of corneal thickness and volume with intraoperative phacoemulsification parameters using Scheimpflug imaging and optical coherence tomography. Journal of Cataract \& Refractive Surgery, 40 (12): 2067-2075.

27. Kemer O, Asfuroglu Y, Kemer O (2019): Central corneal thickness and corneal volume changes in eyes with and without pseudoexfoliation after uneventful phacoemulsification. International Ophthalmology, 39 (2): 275-280.

28. Lundberg B, Jonsson M, Behndig A (2005): Postoperative corneal swelling correlates strongly to corneal endothelial cell loss after phacoemulsification cataract surgery. American Journal of Ophthalmology, 139 (6): 1035-1041.

29. Altay Y, Ozgur B, Gülizar D et al. (2016): Agreement between Corneal Thickness Measurements Using Pentacam Scheimpflug Camera, Noncontact Specular Microscopy, and Ultrasonographic Pachymetry in Diabetic Patients. Current Eye Research, 42: 1-8. 